\title{
Anisotropy of Electrical Transport and Superconductivity in Metal Chains of $\mathrm{Nb}_{2} \mathrm{Se}_{3}$
}

\author{
Rongwei $\mathrm{Hu}^{1,2}$, K. Lauritch-Kullas ${ }^{3}$, J. O'Brian ${ }^{3}$, V. F. Mitrovic ${ }^{2}$ and C. Petrovic ${ }^{1}$ \\ ${ }^{1}$ Condensed Matter Physics, Brookhaven National Laboratory, Upton New York 11973-5000 USA \\ ${ }^{2}$ Physics Department, Brown University, Providence RI 02912 \\ ${ }^{3}$ Quantum Design, 6235 Lusk Blvd., San Diego, CA 92121
}

(Dated: July 18, 2021)

\begin{abstract}
In this work we have shown bulk superconductivity and studied the anisotropy in both the normal and superconducting states in quasi-1D conductor $\mathrm{Nb}_{2} \mathrm{Se}_{3}$. Electron - electron Umklapp scattering dominates electronic transport along the direction of $\mathrm{Nb}$ metal chains as well as perpendicular to it. The superconducting state is rather anisotropic with possible multi - band features.
\end{abstract}

PACS numbers: 74.70.-b, 74.25.Bt, 74.62Bf

\section{INTRODUCTION}

In the recent years, there has been continuing interest in search, the discovery and characterization of materials that exhibit exotic collective electronic phenomena in different bonding and structure types. High $\mathrm{T}_{C}$ cuprates, $\mathrm{Sr}_{2} \mathrm{RuO}_{4}$, some organic and heavy fermion metals are examples of materials which exhibit low dimensional superconductivity gaps or anisotropic Fermi surfaces $\underline{1}^{-4}$ The true character of anisotropy is one of the most important questions to be addressed and in that context quasi$1 \mathrm{D}$ materials are the extreme examples in nature $\underline{5}^{-8}$ Transition metal chalcogenides often host quasi-one dimensional conducting electrons due to existence of metal chains in their crystal structure, where band dispersion along the chain is an order of magnitude larger than dispersion in the direction perpendicular to chains. Intermetallic phases on the selenium-rich side of Nb-Se phase diagram, such as $\mathrm{NbSe}_{2}$ and $\mathrm{NbSe}_{3}$ are fruitful model materials for the study of low dimensional superconductivity and charge density waves (CDW) $\stackrel{9}{2}, 10$ On the other hand, the niobium rich side of the Nb-Se phase diagram has been far less explored, with exception of $\mathrm{Nb}_{3} \mathrm{Se}_{4}$ which is a superconductor with $\mathrm{T}_{C}=2.31 \mathrm{~K} .11$ In this work we show bulk superconductivity and examine the character of anisotropy in the normal and superconducting states of $\mathrm{Nb}_{2} \mathrm{Se}_{3}{ }^{12}$, a quasi-1D conductor whose normal state electronic transport is dominated by electron - electron Umklapp scattering. 13

\section{CRYSTAL STRUCTURE}

$\mathrm{Nb}_{2} \mathrm{Se}_{3}$ crystallizes in the monoclinic $\mathrm{P}_{21 / m}$ crystal structure where $\mathrm{Nb}$ atoms form two types of infinite metal - metal chains running in the b-axis direction: $\mathrm{Nb}(1)$ and $\mathrm{Nb}(2)$. Interatomic distances in the $\mathrm{Nb}(1)$ chain are comparable to those in pure metal $(2.97 \AA)$ whereas metal distances in the $\mathrm{Nb}(2)$ chain are somewhat longer $(3.13 \AA){ }^{14}$ The origin of metal clustering in this crystal structure is due to its main building block, $\mathrm{M}_{2} \mathrm{X}_{6}$ chains (Fig. 1) which are present in many $\mathrm{M}_{2} \mathrm{X}_{3}$ metal - clustering transition metal chalcogenides $(\mathrm{M}=\mathrm{Mo}$, Se, $\mathrm{Ta} ; \mathrm{X}=\mathrm{S}, \mathrm{Se}) \stackrel{15}{=} \mathrm{M}_{2} \mathrm{X}_{6}$ chains are formed by double edge
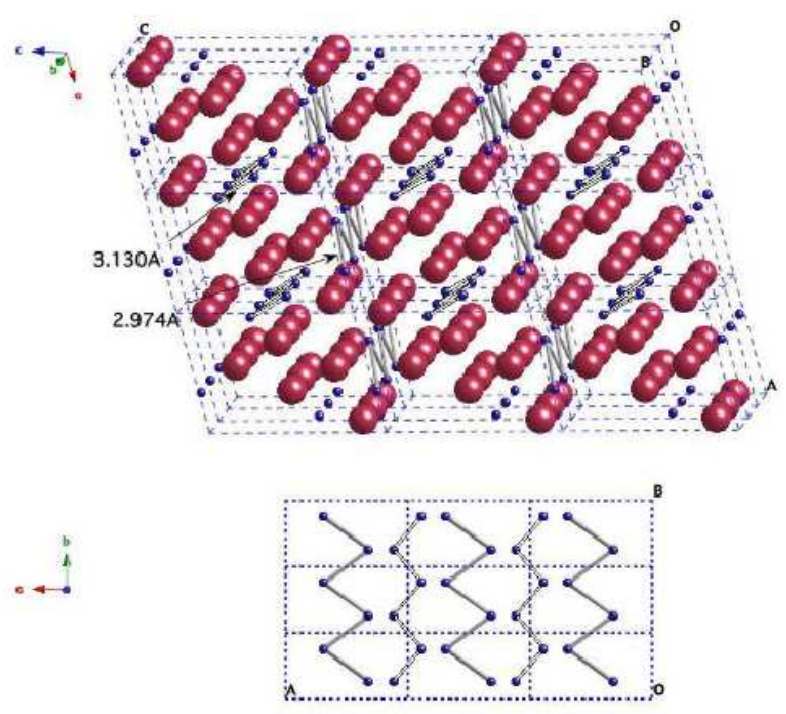

FIG. 1: Crystal structure of $\mathrm{Nb}_{2} \mathrm{Se}_{3}$ (3x3 unit cells shown) and projection onto ab plane showing two types of $\mathrm{Nb}$ chains, open and solid bonds. $\mathrm{Nb}$ (blue symbol), $\mathrm{Sb}$ (red symbol).

sharing of $\mathrm{MX}_{4}$ chains which are in turn formed by edge sharing of ideal $\mathrm{MX}_{6}$ octahedra. Metal - metal bond formation across the shared octahedral edge in $\mathrm{MX}_{4}$ - type chains causes formation of $\mathrm{Nb}(2)$ chains and distortion from ideal octahedral building blocks in $\mathrm{Nb}_{2} \mathrm{Se}_{6}$ clusters. Electrical transport properties and potential structural instabilities in these systems are governed by the bands formed from the set of unfilled $\mathrm{t}_{2 g}$ orbitals.

Due to metal clustering in the basic $\mathrm{Nb}_{2} \mathrm{Se}_{6}$ building blocks band structure is comprised of flat bands originating from $\mathrm{Nb}(2)$ - $\mathrm{Nb}(2)$ bonding across the shared edge of the $\mathrm{NbSe}_{4}$ chains and rather dispersive bands formed from $\mathrm{x}^{2}-\mathrm{y}^{2}$ orbitals along the $\mathrm{Nb}(1)$ chain. $\mathrm{Nb}_{2} \mathrm{Se}_{3}$ is isostructural with $\mathrm{Mo}_{2} \mathrm{~S}_{3}$ where metal - metal distances in both $\mathrm{Mo}(1)$ and $\mathrm{Mo}(2)$ chains are comparable to those in pure Mo metal. Peierls distortion associated with half filled $\mathrm{t}_{2 g}$ block bands due to the oxidation state of Mo atoms $\left[\mathrm{Mo}^{3+}\left(\mathrm{d}^{3}\right)\right]$ doubles the unit cell so $\mathrm{Mo}_{2} \mathrm{~S}_{3}$ exhibits CDW transition. On the other hand, there is no CDW formation in $\mathrm{Nb}_{2} \mathrm{Se}_{3}$ since the oxidation state of 
$\mathrm{Nb}$ atoms is $\mathrm{Nb}^{3+}\left(\mathrm{d}^{2}\right)$. Consequently, $\mathrm{Nb}_{2} \mathrm{Se}_{3}$ is a good model material for the study of low dimensional electronic transport along metallic chains down to the lowest temperatures without partial destruction of the Fermi Surface due to CDW formation, as is the case in many transition metal oxides and chalcogenides.

\section{EXPERIMENT}

Powder X-ray patterns were taken at room temperature using a Rigaku Miniflex with $\mathrm{CuK}_{\alpha}$ radiation. The data were collected using $2 \theta$ scan in the $10^{\circ}-90^{\circ}$ range. Several different single crystals were oriented by a Laue camera. Electrical contacts were made with Epotek H20E silver epoxy for current along the b-axis of the crystal as well as perpendicular to the $b$ axis in the a-c plane (parallel and perpendicular to $\mathrm{Nb}$ - Nb chains). The electrical resistivity was measured in a Quantum Design PPMS-9 in the temperature range from $0.4 \mathrm{~K}-300 \mathrm{~K}$. and up to $90 \mathrm{kOe}$. The heat capacity was measured using a relaxation technique in the same instrument. The magnetic susceptibility was measured in a Quantum Design MPMS XL-5. The dimensions of the samples were measured by a high precision optical microscope, the Nikon SMZ-800 with $10 \mu \mathrm{m}$ resolution, and average values are presented. Electrical resistivity, magnetic susceptibility and heat capacity was reproduced on several independently grown samples from different batches in order to exclude sample dependence.

\section{RESULTS}

The synthesis of large single crystals allowed us to study the anisotropy in the normal and superconducting state of $\mathrm{Nb}_{2} \mathrm{Se}_{3}$. Single crystals of $\mathrm{Nb}_{2} \mathrm{Se}_{3}$ were grown using a molten metallic flux technique, thus avoiding possible contamination and intercalation of transport agent atoms. $16,17,18$ Crystals grew as thin platelike rods with the long rod axis being the b-axis of the crystal structure along the $\mathrm{Nb}-\mathrm{Nb}$ metal chains. Crystal structure parameters of flux grown $\mathrm{Nb}_{2} \mathrm{Se}_{3}$ crystals are in good agreement with previously published: $\mathrm{a}=5.5051(2) \AA, \mathrm{b}=3.4349(2) \AA, \mathrm{c}=9.2369(4) \AA$ and monoclinic angle $\beta=130.16(1)^{\circ}$. The anisotropy in electrical transport for current applied both along and perpendicular to the chain direction at high temperatures is shown in Fig. 2 (a). The resistivity of our flux grown samples for current $\mathrm{I} \uparrow \uparrow \mathrm{b}$ - axis $\left(\rho_{P}\right)$ is in good agreement with the data from crystals obtained using a vapor transport technique. 13 Electrical transport for the current perpendicular to the chains, $\mathrm{I} \perp \mathrm{b}$-axis $\left(\rho_{N}\right)$, is up to an order of magnitude larger, implying less band structure anisotropy than in other linear chain inorganic materials and probably less difference in the band dispersion energy parallel to chains (b - axis) and perpendicular to it 19,20 The resistivity decreases with decrease in temperature
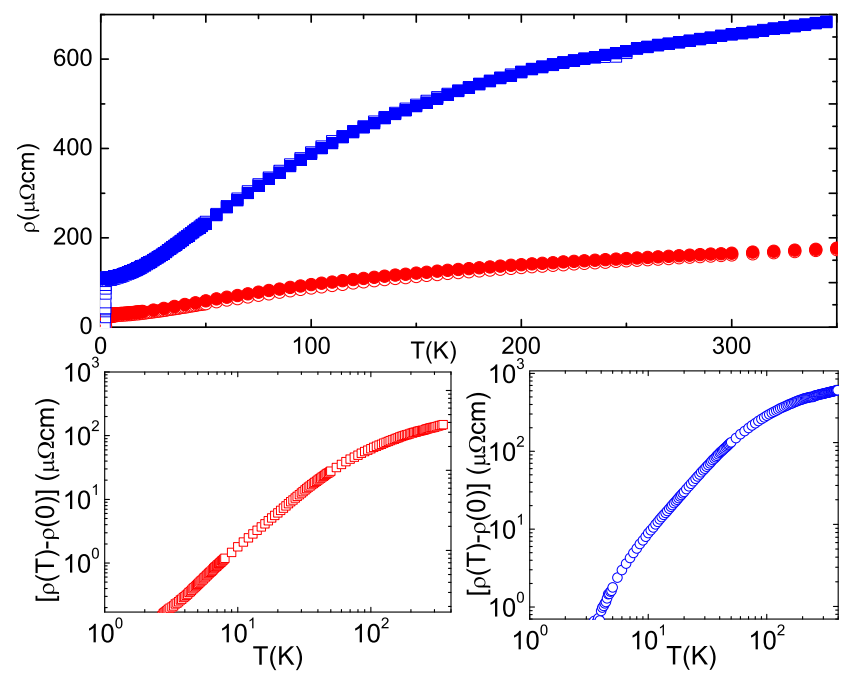

FIG. 2: (a) Electrical transport for the current applied parallel (red) and perpendicular to $\mathrm{Nb}$ chains (b - axis of the crystal structure) of $\mathrm{Nb}_{2} \mathrm{Se}_{3}$. (b) $\rho-\rho_{0}(\mathrm{~T})$ for current applied parallel (left) and perpendicular to $\mathrm{Nb}$ chains (right)

approaching residual resistivity values below $4 \mathrm{~K}, \rho_{P}=$ $26 \mu \Omega \mathrm{cm}$ and $\rho_{N}=105 \mu \Omega \mathrm{cm}$. Fig. 1(b) and 1(c) show the temperature dependence of the resistivity after subtracting the residual resistivity that has been estimated by the extrapolation of the $\rho_{P}$ and $\rho_{N}$ curves to $\mathrm{T}=0$.

The clustering of atoms in chains results in the quasi - one dimensional conduction band model first proposed by Kamimura on the example of $(\mathrm{SN})_{x}, \underline{13}, \underline{21}$ It was shown that electron - electron Umklapp scattering dominates the electronic transport, whereas electron - phonon and electron - electron normal scattering are negligible $\stackrel{22}{\underline{2}}$ The temperature dependence of the resistivity is given by the power law $\rho-\rho_{0}=C T^{n}(\mathrm{C}=$ const. $) \quad$ For $k_{B} T \leq$ $(0.1-0.3) \gamma$, where $\gamma$ is the interchain interaction energy, $n$ takes values of $2 \leq n \leq 3$, and for $k_{B} T>|\gamma|, n=1$. The power law fit of both $\rho_{P}$ and $\rho_{N}$ in Fig. 2(b) is possible below $T=15 K$ whereas a linear temperature dependence is observed above $T=250 \mathrm{~K}$. The resistivity for I $\uparrow \uparrow \mathrm{b}$ - axis takes the form $\rho=\rho_{0}+C T^{n}$ with $n=2.1 \pm 0.1$, $C=(1.40 \pm 0.02) \times 10^{-8} \Omega \mathrm{cm} / K^{n}$ and resistivity for $\mathrm{I} \perp \mathrm{b}$ - axis has the same temperature dependence with $n=2.07 \pm 0.04, C=(7.1 \pm 0.7) \times 10^{-8} \Omega \mathrm{cm} / K^{n}$.

Low temperature thermodynamic, magnetic and transport properties are shown in Fig. 3. The jump $\Delta C$ at $T_{C}=2.0 \mathrm{~K}$ in the specific heat and $25 \%$ of unsaturated $-1 / 4 \pi$ value in $\mathrm{M} / \mathrm{H}$ data at the lowest temperature of our measurement $(1.8 \mathrm{~K})$ suggest a bulk superconducting transition in $\mathrm{Nb}_{2} \mathrm{Se}_{3}$. Electrical transport measurements show a somewhat higher transition temperature. For current applied along the chains as well as perpendicular to chains (b -axis) the onset of the superconducting transition is at $T_{\rho}^{\text {onset }}=2.4 K$. At $T=2.2 \mathrm{~K}$ the transition to the superconducting state 


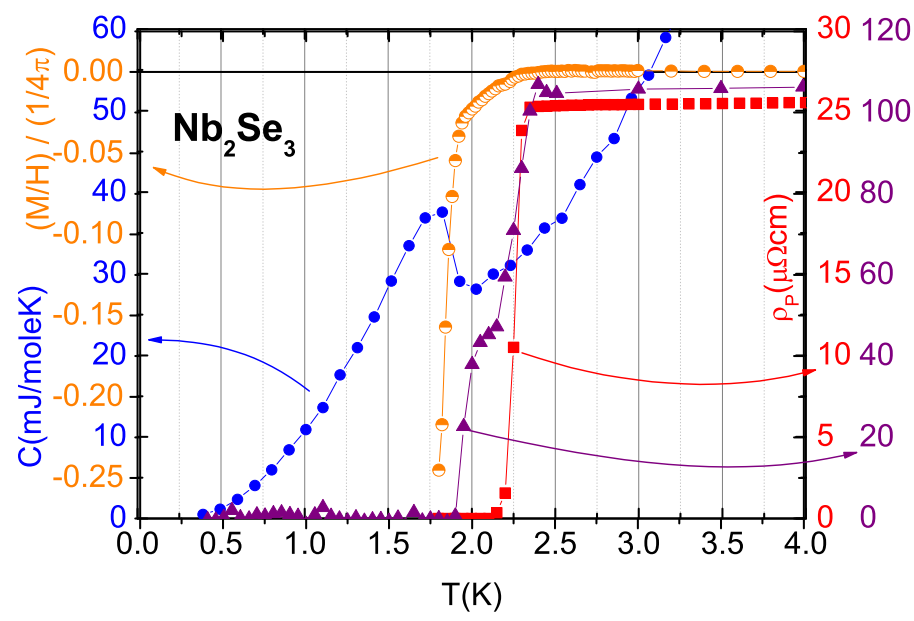

FIG. 3: Superconductivity in $\mathrm{Nb}_{2} \mathrm{Se}_{3}$. Left axis shows thermodynamic properties (heat capacity and magnetization) and right axis shows electrical transport properties for current applied parallel (red symbols) and perpendicular (violet symbols) to $\mathrm{Nb}$ chains (b - axis of the unit cell)

for the $\mathrm{b}$ - axis $\left(\rho_{P}\right)$ resistivity is complete whereas resistivity for $\mathrm{I} \perp \mathrm{b}$ - axis $\left(\rho_{N}\right)$ shows a structure and the change of slope. Finally, at bulk $T_{C}=2.0 \mathrm{~K} \rho_{N}$ is fully superconducting. Magnetic susceptibility $\mathrm{M} / \mathrm{H}$ is diamagnetic already at $\mathrm{T}_{\rho}^{\text {onset }}$ and its magnitude grows towards bulk superconductivity below $\mathrm{T}_{C}=2.0 \mathrm{~K}$. By fitting the temperature dependence of the specific heat in the normal state using $C=\gamma T+\beta T^{3}$ we obtain $C / T=\gamma=9.96 \pm 0.2 \mathrm{~mJ} / \mathrm{moleK}^{2}$ and $\theta_{D}=223 \pm 3 \mathrm{~K}$ using the relation $\theta_{D}^{3}=(12 / 5)\left(\pi^{4} n R / \beta\right)$, where $\mathrm{R}$ is the gas constant and $n$ is the number of atoms per molecule.

Figure 4 presents temperature-dependent electrical resistivity data for $\mathrm{Nb}_{2} \mathrm{Se}_{3}$ taken at a variety of applied fields for $\mathrm{H} \preceq 12 \mathrm{kOe}$ for field applied parallel and perpendicular to $\mathrm{Nb}$ chains and for the current running parallel and perpendicular to $\mathrm{Nb}$ chains. Two features are evident: there is a suppression of the superconducting phase to lower temperatures for increasing applied field, and there is a negligible magnetoresistivity in the normal state. The decrease of $\mathrm{T}_{C}$ for $\mathrm{I} \uparrow \uparrow \mathrm{b}$ - axis tracks well the data for $\mathrm{I} \perp \mathrm{b}$ - axis for a fixed field direction. On the other hand, suppression of superconductivity is much stronger for $\mathrm{H} \perp \mathrm{b}$ axis ( $\mathrm{Nb}$ chains) than for a field applied along b- axis (parallel to $\mathrm{Nb}$ chains). A closer look at the temperature dependent resistivity for I $\perp$ b axis reveals a step in the superconducting transition that persists up to $1 \mathrm{kOe}$. Using these data, $\mathrm{H}_{c 2}(\mathrm{~T})$ curve can be deduced (Fig. 5). We notice an almost linear temperature dependence and relatively large anisotropy of the $\mathrm{H}_{c 2}(\mathrm{~T})$ curve for a magnetic field applied parallel and perpendicular to the $\mathrm{Nb}$ chains. There is no sign of saturation down to the lowest temperature of our measurement, $0.4 \mathrm{~K}$.
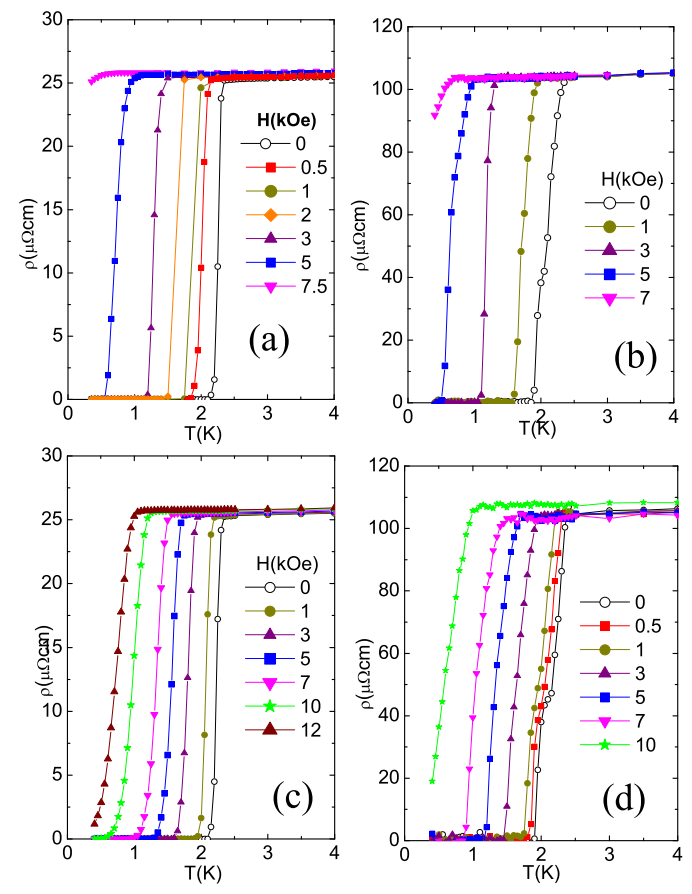

FIG. 4: Temperature dependant resistivity for field applied perpendicular $(\mathrm{a}, \mathrm{b})$ and parallel to $\mathrm{Nb}$ chains $(\mathrm{b}, \mathrm{d})$, with current applied parallel $(\mathrm{a}, \mathrm{c})$ and perpendicular $(\mathrm{b}, \mathrm{d})$ to $\mathrm{Nb}$ chains.

By extrapolating $\mathrm{H}_{c 2}(\mathrm{~T})$ data to $\mathrm{T}=0$ we get values of $\mathrm{H}_{c 2}(0)=7.5 \mathrm{kOe}(\mathrm{H} \perp \mathrm{Nb}$ chains $)$ and $\mathrm{H}_{c 2}(0)=14$ $\mathrm{kOe}(\mathrm{H} \uparrow \uparrow \mathrm{Nb}$ chains $)$, which is significantly larger. Taken as a whole, the temperature dependence of $\mathrm{H}_{c 2}$ for $\mathrm{Nb}_{2} \mathrm{Se}_{3}$ is similar to that found for other quasi one dimensional superconductors, for example $\mathrm{TaSe}_{3}, \mathrm{Nb}_{3} \mathrm{Se}_{4}$ and $\mathrm{Nb}_{3} \mathrm{~S}_{4}$. By using $\mathrm{B}_{C 2}(\mathrm{~T})=[\Phi 0 / 2 \pi \xi(\mathrm{T})]$ we obtain coherence lengths $\xi(0)=203 \AA, \xi(0)=153 \AA$ for a field applied perpendicular and parallel to $\mathrm{Nb}$ chains, respectively, a bit shorter than in $\mathrm{Nb}$ metal $\xi(0)=380 \AA$.

\section{DISCUSSION}

Electronic transport in the normal state can be understood in the framework of the theory of Oshiyama and Kamimura $\stackrel{22}{\underline{2}}$ However, our results imply that electron electron Umklapp scattering is dominant not only along the chain axis of a quasi-one-dimensional metal but also perpendicular to it. The linear resistivity above $250 \mathrm{~K}$ and a $\rho \sim \mathrm{T}^{2.1}$ temperature dependence of resistivity for both $\rho_{P}$ and $\rho_{N}$ below $\mathrm{T}=15 \mathrm{~K}$, are consistent with the possible range of the chain interaction energy $45 \mathrm{~K}$ $\preceq \gamma \preceq 150 \mathrm{~K}$. We note that the power law temperature dependence of resistivity in vapor transport grown crystals extends up to $40 \mathrm{~K}$, implying a larger value of $\gamma$ than what we obtained in our work on flux grown crystals. The difference could arise due to the presence of 


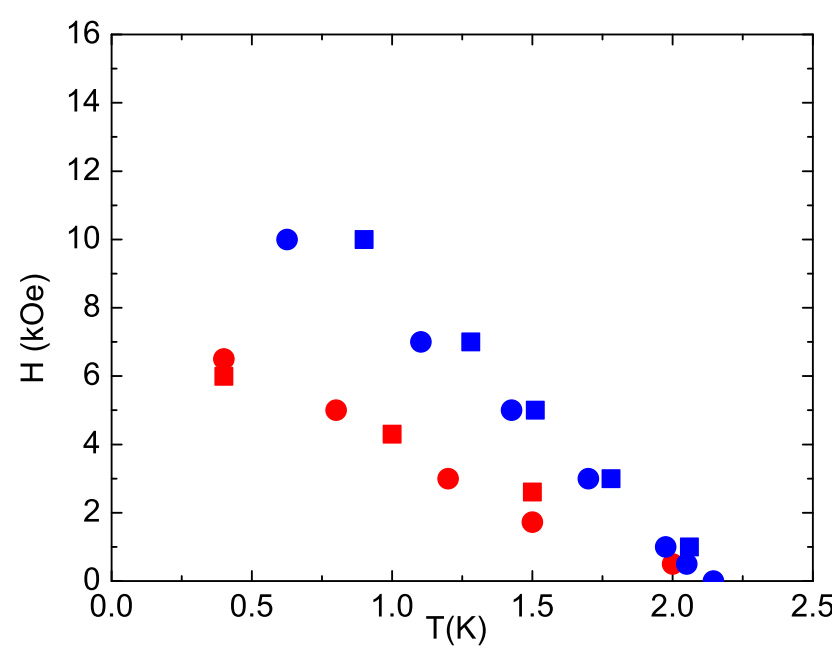

FIG. 5: $\mathrm{H}_{c 2}$ (T) for fields applied perpendicular (red) and parallel to Nb chains (blue symbols) with current applied perpendicular (circles) and parallel to $\mathrm{Nb}$ chains (squares).

other scattering mechanisms besides electron - electron Umklapp scattering. Alternatively, this may imply that the interchain interaction energy is sensitive to crystalline disorder and imperfections. The flux grown crystals have a higher residual resistivity value $\rho_{0}=26 \mu \Omega \mathrm{cm}$ for the current applied parallel to $\mathrm{Nb}$ chains along $\mathrm{b}$-axis of the crystal compared to crystals grown by vapor transport reaction where $\rho_{0}=0.5 \mu \Omega \mathrm{cm} . \underline{13}$

We turn now to the properties of the superconducting state. Using McMillan's expression 23

$$
T_{C}=\frac{\Theta_{D}}{1.45} \exp \left\{-\frac{1.04(1+\lambda)}{\lambda-\mu^{*}(1+0.62 \lambda)}\right\}
$$

and value of Debye temperature from the heat capacity analysis $\theta_{D}=223 \mathrm{~K}$, we estimate the value of the electron - phonon coupling constant $\lambda=1.05$ assuming the empirical value of pseudopotential $\mu^{*}=0.1$. These results puts $\mathrm{Nb}_{2} \mathrm{Se}_{3}$ in the class of the intermediate to strong coupling superconductors. The specific-heat jump $\Delta \mathrm{C} /\left(\gamma \mathrm{T}_{C}\right) \approx$ 0.5 (Fig. 3) is substantially smaller than the mean - field BCS value of $1.43 \stackrel{24}{2}$ In the weak-coupling superconductors, the reduced specific heat jump can be interpreted in the terms of the energy gap anisotropy. The effects of an anisotropic energy gap on the thermodynamic properties of the BCS superconductors have been calculated in the seminal work of John Clemm:

$$
\Delta C /\left(\gamma T_{C}\right)=1.426\left(1-4\left\langle a^{2}\right\rangle\right)
$$

where $\left\langle a^{2}\right\rangle$, the mean-squared anisotropy, is the average over the Fermi surface of the square of the deviation of the energy-gap parameter from its average ${ }^{25}$ Strong coupling effects and anisotopy in general tend to work in opposite direction and the specific heat jump would be enhanced by strong coupling. This would imply a rather strong anisotropy of the superconducting gap in $\mathrm{Nb}_{2} \mathrm{Se}_{3}$. Equation (2) therefore gives a rather conservative estimate of $\left\langle a^{2}\right\rangle=0.16$. Table 1 compares the value of electron phonon coupling parameter and the gap anisotropy for several anisotropic superconductors.

$\mathrm{Nb}_{2} \mathrm{Se}_{3}$ exhibits zero resistivity for current applied along the $\mathrm{b}$ - axis of the crystal, parallel to $\mathrm{Nb}$ chains $\left(\rho_{P}\right)$ at $\mathrm{T}=2.2 \mathrm{~K}$, whereas the onset of this transition and the transition to a diamagnetic $\mathrm{M} / \mathrm{H}$ is at $\mathrm{T}_{\rho}^{\text {onset }}$ $=2.4 \mathrm{~K}$. However, the heat capacity shows a transition to a bulk superconducting state at bulk $\mathrm{T}_{C}=2.0$ $\mathrm{K}$, where magnetic susceptibility data for $\mathrm{M} / \mathrm{H}$ shows a dive towards full Meisner effect. These results suggest that the superconductivity of $\mathrm{Nb}_{2} \mathrm{Se}_{3}$ is a bulk effect below $\mathrm{T}_{C} \sim 2.0 \mathrm{~K}$ and filamentary in nature between $\mathrm{T}_{\rho}^{\text {onset }}$ and $\mathrm{T}_{C} . \mathrm{Nb}_{2} \mathrm{Se}_{3}$ can be regarded as an aggregate of one-dimensional chains that are weakly coupled through Josephson-type junctions since the distance between the $\mathrm{Nb}$ atoms in a chain is metallic but the interchain distance exceeds the metallic distance. The temperature dependence of electrical resistivity for current $\mathrm{I} \perp \mathrm{b}-$ axis $\left(\rho_{N}\right.$ - perpendicular to Nb chains) is consistent with this interpretation. The onset temperature $\mathrm{T}_{\rho}^{o n s e t}$ is the same for both $\rho_{P}$ and $\rho_{N}$, however $\rho_{N}$ shows zero resistivity only at bulk $\mathrm{T}_{C}$ and a feature indicating another superconducting transition at $\mathrm{T}=2.2$ $\mathrm{K}$ that is visible in applied magnetic field up to $1 \mathrm{kOe}$. An alternative explanation for this is a small misorientation in the current direction during electrical transport measurement perpendicular to the $\mathrm{Nb}$ chains. However that would imply the presence of two $\mathrm{T}_{C}$ 's in two different electronic substructures and negligible interband scattering, $\underline{26}$

Table 1: Fundamental parameters of the superconducting state of $\mathrm{Nb}-\mathrm{Se}$ and $\mathrm{Nb}-\mathrm{S}$ superconductors. Data on $\mathrm{Nb}$ is given for comparison.

\begin{tabular}{||c||c||c||c||}
\hline \hline & $\mathrm{T}_{C}(\mathrm{~K})$ & $\left\langle a^{2}\right\rangle$ & $\lambda$ \\
\hline \hline $\mathrm{NbSe}_{2}$ & 7.1 & 0.04 & 0.30 \\
\hline \hline $\mathrm{Nb}_{3} \mathrm{~S}_{4}$ & 3.78 & 0.07 & 0.51 \\
\hline \hline $\mathrm{Nb}_{3} \mathrm{Se}_{4}$ & 2.31 & 0.15 & 0.51 \\
\hline \hline $\mathrm{Nb}_{2} \mathrm{Se}_{3}$ & 2.0 & 0.16 & 1.05 \\
\hline \hline $\mathrm{Nb}$ & 9.25 & 0.01 & 1 \\
\hline \hline
\end{tabular}

\section{CONCLUSION}

In summary, we have showed bulk superconductivity, and have investigated the anisotropy in electrical transport properties in the normal and in the superconducting state of $\mathrm{Nb}_{2} \mathrm{Se}_{3}$. Our results show that $\mathrm{Nb}_{2} \mathrm{Se}_{3}$ is in the intermediate to large coupling limit of BCS theory with 
a possible large mean - squared anisotropy of the energy gap on the Fermi surface.

More microscopic measurements such as NMR, neutron scattering and tunneling experiments would be very useful to quantify the question of possible filamentary superconducitivity above bulk $\mathrm{T}_{C}$ or multiband features. We conclude that the $\mathrm{Nb}_{2} \mathrm{Se}_{3}$ is a promising model sys- tem to study superconducting properties in quasi one dimensional metallic chain systems.

We thank S. L. Bud'ko, P. C. Canfield, M. Strongin and Z. Fisk for useful discussions. This work was carried out at the Brookhaven National Laboratory which is operated for the U.S. Department of Energy by Brookhaven Science Associates (DE-Ac02-98CH10886).
1 J.G. Bednorz and K.A. Muller, "Possible High Tc Superconductivity in the Ba-La-Cu-O System," Z. Phys.B 64, 189 (1986)

2 Y. Maeno, H. Hashimoto, K. Yoshida, S. Nishizaki, T. Fujita, J. G. Bednorz, F. Lichtenberg Nature 372, 532 (1994)

3 T. Ishiguro, K. Yamaji, and G. Saito, Organic Superconductors, 2nd ed. (Springer, Berlin, 1998)

${ }^{4}$ C. Petrovic, P. G. Pagliuso, M.F. Hundley, R. Movshovich, J.L. Sarrao, J. D. Thompson, Z. Fisk and P. Monthoux, J. Phys.: Cond. Matter Lett.13, L337 (2001)

${ }^{5}$ R. C. Morris, R. V. Coleman and R. Bhandari, Phys. Rev. B 5, 895 (1972)

${ }^{6}$ N. Kaiser and J. Silk, Nature (London) 324, 529 (1986)

7 I. C. McManus, Nature (London) 259, 426 (1976)

8 S. L. Bud'ko, V. G. Kogan and P. C. Canfield,Phys. Rev. B 64, 180506

9 T. Yokoya, T. Kiss, A. Chainani, S. Shin, M. Nohara and H. Takagi Science 294, 2518 (2001)

10 A. Perucchi, L. Degiorgi and R. E. Thorne, Phys. Rev. B 69, 195114 (2004)

11 T. Ishida, K. Kanoda, H. Mazaki and I. Nakada, Phys. Rev. B 29, 1183 (1984)

12 K. Igaki and S. Nishine, J. Japan Inst. Metalas 41, 843 (1977)

13 M. H. Rashid and D. J. Sellmyer, Phys. Rev. B 29, 2359
(1984)

14 F. Kadijk, R. Huisman and F.Jelinek, Acta Cryst. B24, 1102 (1968)

15 E. Canadell, A. LeBeuze, M. A. El Khalifa, R. Chevrel, and Myung-Hwan Whangbo, J. Am. Chem. Soc 111, 3778 (1989)

16 Z. Fisk, J.P. Remeika, in: K.A. Gschneider, J. Eyring (Eds.), Handbook on the Physics and Chemistry of Rare Earths, Vol.12, Elsevier, Amsterdam, 1989.

17 P.C. Canfield, Z. Fisk. Phil. Mag. B 65, 1117 (1992)

18 P.C. Canfield, I.R. Fisher, J. Cryst. Growth 225, 155 (2001)

19 A. Oshiyama, J. Phys. Soc. Jpn. 52, 587 (1983)

20 A. Oshiyama, Solid State Commun. 43, 607 (1982)

21 H. Kamimura, A. M. Glazer, A. J. Grant, J. Natsume, M. Schreiber and A. D. Yoffe, J. Phys. C 9,291 (1976)

22 A. Oshiyama and K. Kamimura, J. Phys. C 14, 5091 (1981)

23 W. L. McMillan, Phys. Rev. 167, 331 (1968)

24 J. Bardeen, L. N. Cooper, and J. R. Schrieffer, Phys. Rev. 108, 1175 (1957)

25 J. R. Clem, Ann. Phys. 40, 268 (1966)

26 H. Suhl, B. T. Matthias and L. R. Walker, Phys. Rev. Lett. 3 (1959) 